\title{
Reviewers for 2019
}

Ghana Med J 2020; 54(1): 1-2 DOI: http://dx.doi.org/10.4314/gmj.v54i1.1

Reviewers of manuscripts for our journal contribute immensely to the standard and quality of the papers we publish.
The Ghana Medical Journal is grateful for the voluntary service provided by the following reviewers for the year 2019.

\begin{tabular}{|c|c|c|c|c|c|}
\hline Abdulai, Alhassan & Ghana & Asuquo, Marcus & Nigeria & Kazembe, Lawrence & $\mathrm{Na}-$ \\
\hline Abiola, Abdul-Hakeem & Nigeria & Atta, Dzifa Abla & Ghana & mibia & \\
\hline Aboagye, Anthony & Ghana & Baah, Winfred & Ghana & Kee, Chee Cheong & Malay- \\
\hline Abuosi, Aaron & Ghana & Baduku, Tokan & Nigeria & sia & \\
\hline Acquah, Evelyn & Ghana & Baidoo, Kenneth & Ghana & Kenu, Ernest & Ghana \\
\hline Adabayeri, Victoria May & Ghana & \multicolumn{2}{|l|}{ Bediako-Bowan, Antoinette } & Kitcher, Emmanuel & Ghana \\
\hline Adanikin, Abiodun & Nigeria & & Ghana & Kokuro, Collins & Ghana \\
\hline Addo, Edwina Beryl & Ghana & Bello, Ajediran & Ghana & Koram, Kwadwo & Ghana \\
\hline Adeniran, Abiodun & Nigeria & Biritwum, Richard & Ghana & Kudah, Catherine & Ghana \\
\hline Adokiya, Martin & Ghana & Boakye, Hosea & Ghana & Kwakyi, Edward & Ghana \\
\hline Adu-Aryee, Nii-Armah & Ghana & Boima, Vincent & Ghana & Kyei, Mathew & Ghana \\
\hline Afriyie-Mensah, Jane & Ghana & Bonavina, Luigi & Italy & Lamptey, Roberta & Ghana \\
\hline Afriyie, James & Ghana & Bonney, Joseph & Ghana & Lartey, Margaret & Ghana \\
\hline Agbenorku, Pius & Ghana & Bonsu, Frank & Ghana & Lokpo, Sylvester & Ghana \\
\hline Agongo, Erasmus & Ghana & Braimah, Ramat & Nigeria & Malm, Keziah & Ghana \\
\hline Agwu, Ezera & Uganda & Busija, Lucy & Ghana & Mensah, Kwesi & Ghana \\
\hline Agyei-Nkansah, Adwoa & Ghana & Cannon, Stephen C. & United & Merga, Hailu & Ghana \\
\hline Agyekum, Francis & Ghana & States & & Michael, Godpower & Nigeria \\
\hline Ahmed, Kofi & Ghana & da-Costa Vroom, Frances & Ghana & Ming, L C & Ghana \\
\hline Ahorlu, Collins & Ghana & Dakubo, Jonathan & Ghana & Moffatt, Cameron & Aus- \\
\hline Aikins, Moses & Ghana & Darko, Rudolph & Ghana & tralia & \\
\hline Ajayi, Isaac & Ghana & De Souza, Dziedzom & Ghana & Mofon, Chukwuemeke & Nigeria \\
\hline Akakpo, Patrick & Ghana & Dei-Adomakoh, Yvonne & Ghana & Motilewa, Olugbemi & Nigeria \\
\hline Akase, Iorhen & Nigeria & Desalu, Olufemi & Nigeria & Newman, Mercy & Ghana \\
\hline Akinmoladun, Janet & Nigeria & Dogbe, Edith & Ghana & Nkrumah, Kofi & Ghana \\
\hline Akpalu, Josephine & Ghana & Dornoo, Bernard & Ghana & Nkyekyer, Kobina & Ghana \\
\hline Al-Aubaidy, Hayder & Aus- & Duah, Amoako & Ghana & Nonvignon, Justice & Ghana \\
\hline tralia & & Edwin, Frank & Ghana & Nottidge, Bolanle & Nigeria \\
\hline Al-Worafi, Yaser & UAE & Ekem, Ivy & Ghana & Nsaful, Josephine & Ghana \\
\hline Alfotawi, Randa A & Ghana & Eliason, Sebastian & Ghana & Nsiah, Kwabena & Ghana \\
\hline Amable, Eugene & Ghana & Enimil, Anthony & Ghana & Ntekim, Atara & Nigeria \\
\hline Amissah-Arthur, Kwesi & Ghana & Enos, Juliana & Ghana & Nuertey, Benjamin & Ghana \\
\hline Amoakoh-Coleman, Mary & Ghana & Essuman, Akye & Ghana & Odeniyi, Ifedayo & Nigeria \\
\hline Amofah, George & Ghana & Faried, Kyei & Ghana & Ofori-Adjei, David & Ghana \\
\hline \multicolumn{2}{|l|}{ Amoyaw-Asamoah, Isabella } & Faruk, Abdulmunaf & Nigeria & Ofori-Adjei, Yaw Adjei & Ghana \\
\hline & Ghana & Fiakpornoo, Martina & Ghana & Ogunwale, Akintayo & Nigeria \\
\hline Ampofo, William & Ghana & Forson, Paa & Ghana & Ohene, Sally-Ann & Ghana \\
\hline \multicolumn{2}{|c|}{ Andoh-Adjei, Francis-Xavier } & Ganyaglo, Gabriel & Ghana & Olamoyegun, Michael & Nigeria \\
\hline & Ghana & Hewlett, Sandra & Ghana & Olasode, Olayinka & Nigeria \\
\hline Angmorterh, Seth & Ghana & Hodgson, Abraham & Ghana & Olowookere, Samuel & Nigeria \\
\hline Anim, J Tei & Ghana & Idowu, Ademola & Nigeria & Onyegbutulem, Henry & Nigeria \\
\hline Ansah, Justina & Ghana & Idowu, Bukunmi & Nigeria & Oppong, Samuel & Ghana \\
\hline Antwi, Sampson & Ghana & Isah, Ambrose & Nigeria & Osafo, Chalotte & Ghana \\
\hline Aryeetey, Richmond & Ghana & Isara, Alphonsus & Nigeria & Osei, Akwasi & Ghana \\
\hline Asamoah, Kojo & Ghana & Jalili, Cyrus & Iran & Ossai, Edmund Ndudi & Nigeria \\
\hline Asare Quansah, Gloria & Ghana & Johnson, Ofonime & Nigeria & Otoghile, Bright & Nigeria \\
\hline Asare, J. B & Ghana & & & Owoo, Nkechi & Ghana \\
\hline
\end{tabular}




\begin{tabular}{|c|c|c|c|c|c|}
\hline Owusu, Enid & Ghana & Sackey, Adziri & Ghana & Tetteh, Raymond & Ghana \\
\hline Owusu, Isaac & Ghana & Sackey, Samuel & Ghana & Torpey, Kwame & Ghana \\
\hline Oyewole, Olufemi & Nigeria & Samba, Ali & Ghana & Udofia, Emilia & Ghana \\
\hline Paintsil, Albert & Ghana & Sarkodie, Badu & Ghana & Umuerri, Ejiroghene & Nigeria \\
\hline Puplampu, Peter & Ghana & Shenoy, Suchitra & India & Yawson, Alfred & Ghana \\
\hline Renner, Lorna & Ghana & Sumankuuro, Joshua & Aus- & Yeboah-Manu, Dorothy & Ghana \\
\hline $\begin{array}{l}\text { Rogerson, Stephen } \\
\text { tralia }\end{array}$ & Aus- & $\begin{array}{l}\text { tralia } \\
\text { Tachi, Kenneth }\end{array}$ & Ghana & Yorke, Ernest & Ghana \\
\hline Sabblah, George & Ghana & Taherkhani, Reza & Iran & & \\
\hline
\end{tabular}

\section{Obituary - Professor Rudolph Darko}

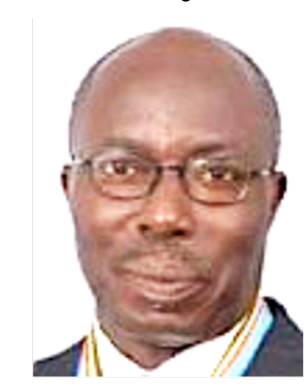

The Publisher and Editorial Board of the Ghana Medical Journal announce with the deepest regret the death of Professor Rudolph Darko on the $23^{\text {rd }}$ February 2020. Professor Darko was appointed a member of the Editorial Board of the Ghana Medical Journal in 1996 and served several terms until his death. In recognition of his services to the Ghana Medical Association, he was elected a Fellow of the Association. He was honoured with Officer of the Order of Volta (Medicine) by the President of the Republic of Ghana in 2008. He joined the University of Ghana Medical School in 1989 and rose to become Associate Professor of Surgery. 